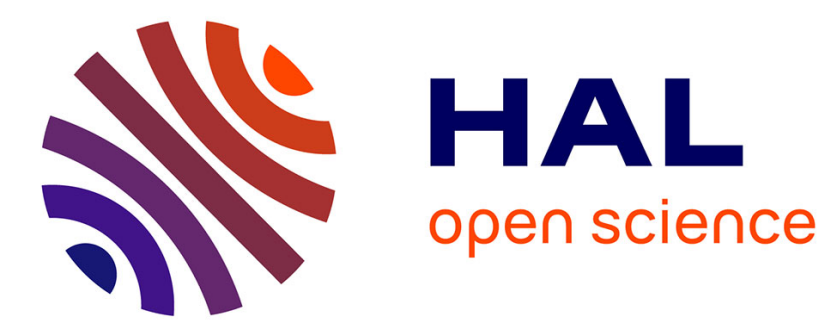

\title{
Synchronized flutter of two slender flags
}

Jérôme Mougel, Olivier Doaré, Sébastien Michelin

\section{To cite this version:}

Jérôme Mougel, Olivier Doaré, Sébastien Michelin. Synchronized flutter of two slender flags. Journal of Fluid Mechanics, 2016, 801, pp.652-669. 10.1017/jfm.2016.451 . hal-01562958

\section{HAL Id: hal-01562958 \\ https://hal.science/hal-01562958}

Submitted on 17 Jul 2017

HAL is a multi-disciplinary open access archive for the deposit and dissemination of scientific research documents, whether they are published or not. The documents may come from teaching and research institutions in France or abroad, or from public or private research centers.
L'archive ouverte pluridisciplinaire HAL, est destinée au dépôt et à la diffusion de documents scientifiques de niveau recherche, publiés ou non, émanant des établissements d'enseignement et de recherche français ou étrangers, des laboratoires publics ou privés. 


\section{OATAO \\ Open Archive Toulouse Archive Ouverte}

Open Archive TOULOUSE Archive Ouverte (OATAO)

OATAO is an open access repository that collects the work of Toulouse researchers and makes it freely available over the web where possible.

This is an author-deposited version published in :

http://oatao.univ-toulouse.fr/

Eprints ID : 18071

To link to this article : DOI:10.1017/jfm.2016.451

URL : http://dx.doi.org/10.1017/jfm.2016.451

To cite this version : Mougel, Jérôme and Doaré, Olivier and Michelin, Sébastien Synchronized flutter of two slender flags. (2016) Journal of Fluid Mechanics, vol. 801. pp. 652-669. ISSN 0022-1120

Any correspondence concerning this service should be sent to the repository administrator: staff-oatao@listes-diff.inp-toulouse.fr 


\title{
Synchronized flutter of two slender flags
}

\author{
Jérôme Mougel $^{1,2, \dagger}$, Olivier Doaré ${ }^{3}$ and Sébastien Michelin ${ }^{1}$ \\ ${ }^{1}$ LadHyX, Département de Mécanique, Ecole Polytechnique - CNRS, 91128 Palaiseau, France \\ ${ }^{2}$ Institut de Mécanique des Fluides de Toulouse, CNRS-UPS-Université de Toulouse, \\ Allée Camille Soula, 31400 Toulouse, France \\ ${ }^{3}$ IMSIA, ENSTA ParisTech, CNRS, CEA, EDF, Université Paris-Saclay, 828 bd des Maréchaux, \\ 91762 Palaiseau, France
}

The interactions and synchronization of two parallel and slender flags in a uniform axial flow are studied in the present paper by generalizing Lighthill's elongated body theory (EBT) and Lighthill's large-amplitude elongated body theory (LAEBT) to account for the hydrodynamic coupling between flags. The proposed method consists of two successive steps, namely the reconstruction of the flow created by a flapping flag within the LAEBT framework and the computation of the fluid force generated by this non-uniform flow on the second flag. In the limit of slender flags in close proximity, we show that the effect of the wakes has little influence on the long-time coupled dynamics and can be neglected in the modelling. This provides a simplified framework extending LAEBT to the coupled dynamics of two flags. Using this simplified model, both linear and large-amplitude results are reported to explore the selection of the flapping regime as well as the dynamical properties of two side-by-side slender flags. Hydrodynamic coupling of the two flags is observed to destabilize the flags for most parameters, and to induce a long-term synchronization of the flags, either in-phase or out-of-phase.

Key words: aerodynamics, flow-structure interactions

\section{Introduction}

A flexible plate or filament may flap spontaneously in a uniform axial flow as a result of the competition between its internal rigidity, its inertia and the destabilizing fluid forces resulting from the deflection of the fluid particles by the deforming structure. This flutter or 'flag' instability and resulting flapping motion have received much interest as exemplified in the recent review of Shelley \& Zhang (2011). Beyond its academic interest or traditional applications, flag flutter has also recently been studied to extract energy from an incoming flow, for example by converting the flapping motion into an electric current using flags covered by electro-active materials (Doaré \& Michelin 2011; Giacomello \& Porfiri 2011).

Understanding the hydrodynamic coupling of multiple flags is critical in this context, in particular, to assess how it affects the flapping properties, synchronization and more generally the collective performance of an assembly of piezoelectric flags. Two-dimensional soap-film experiments by Zhang et al. (2000) on two flexible filaments revealed an in-phase synchronization for small separation distances, and an

$\dagger$ Email address for correspondence: jerome.mougel@imft.fr 
out-of-phase synchronization at larger distances, a trend later supported by further experimental, theoretical and numerical studies (Zhu \& Peskin 2003; Jia et al. 2007). More insight on the two-dimensional multiple-flag dynamics was also gained from experiments (Schouveiler \& Eloy 2009), linear stability analysis (Michelin \& Llewellyn Smith 2009) and numerical simulations (Farnell, David \& Barton 2004; Alben 2009; Tian et al. 2011a).

These studies all focus on the two-dimensional problem which is representative of the three-dimensional case only when the width of the flags is much larger than its length. The coupling of multiple flags for arbitrary span, and in particular for slender flags, remains poorly documented despite its practical importance, for energy harvesting purposes for instance. Direct numerical simulations of the fluid-solid systems are possible (Banerjee, Connell \& Yue 2015), but their complexity and computational cost prohibit at the moment systematic parametric studies or optimization and emphasize the need for reduced-order modelling of these interactions. The main objective of the paper is therefore to provide a simplified model in the slender body limit allowing us both to give insights to the physical synchronization process and to provide a useful benchmark for subsequent works on the topic.

In potential flow, fluid forces on a single flapping slender flag can be computed as a reactive local force: an added momentum $m_{a} \boldsymbol{u}_{n}$ is associated with each slice of fluid normal to the structure's centreline, with $\boldsymbol{u}_{n}$ the local normal relative velocity of the solid with respect to the background flow and $m_{a}$ the added mass coefficient of the structure's cross-section; the reactive force results from changes in the momentum of the fluid advected along the deformed structure. This idea is at the heart of elongated body theory (EBT) (Lighthill 1960) and its generalization to nonlinear flapping dynamics, the large-amplitude elongated body theory (LAEBT) (Lighthill 1971). The powerful advantage of this method is its simplicity: the fluid force is expressed solely in terms of the local kinematics of the solid body. Extensions to this theory have recently been proposed for three-dimensional body motions (Candelier, Boyer \& Leroyer 2011) and weakly non-uniform background flows (Candelier, Porez \& Boyer 2013).

The present article extends this approach to model the flapping dynamics of several slender flags and is organized as follows. Section 2 presents the problem's geometry, the relevant parameters and structural model, and $\S 3$ describes the method for computing the fluid forces in the presence of hydrodynamic interactions based on a generalization of Lighthill's LAEBT to the case of two flags. The linear stability and mode selection of the two-flag configuration is analysed in $\S \S 4$ and 5 focusing on flag synchronization in the saturated large-amplitude dynamics. Finally, $\S 6$ proposes a discussion of the problem and new opportunities for future work.

\section{Problem setting}

We consider two parallel, rectangular and infinitely thin flags of length $L$ and width $H$ in a steady uniform flow of velocity $U_{\infty}$, density $\rho$ and kinematic viscosity $\nu$, with $d$ the distance between their clamped leading edges (figure 1a). Both flags are inextensible, with surface density $\mu$ and bending rigidity $B$; gravity is neglected. When $R e=U_{\infty} L / v \gg 1$, viscous effects are negligible except within thin boundary layers around the flags that separate at the trailing edge into vortex sheets; thus, a potential flow model is used. The flag and flow dynamics are both governed by four nondimensional parameters, namely

$$
H^{*}=\frac{H}{L}, \quad d^{*}=\frac{d}{L}, \quad M^{*}=\frac{\rho L}{\mu}, \quad U^{*}=U_{\infty} L \sqrt{\frac{\mu H}{B}},
$$


(a)

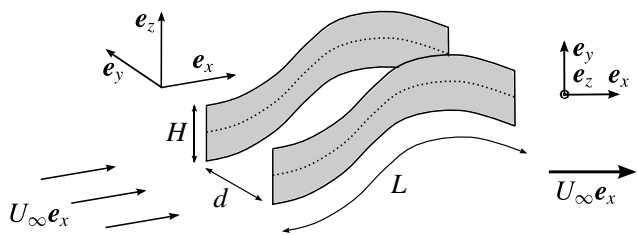

(b)

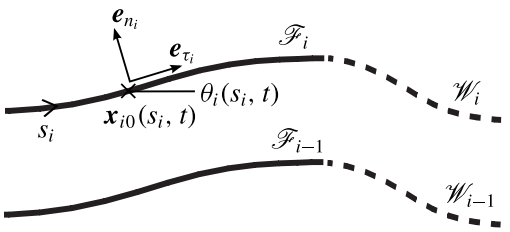

FIGURE 1. Sketches of the configuration: $(a)$ three-dimensional view, $(b)$ view from above.

which respectively correspond to the flags' aspect ratio, the non-dimensional inter-plate distance, the mass ratio and the reduced velocity.

In the following, $\mathscr{F}_{i}$ and $\mathscr{W}_{i}$ denote the $i$ th flag and its wake $(i=1,2)$. Neglecting streamwise torsion and spanwise displacement, the position $\boldsymbol{x}_{i}\left(s_{i}, z_{i}, t\right)$ of $\mathscr{F}_{i}$ is entirely described by its centreline position $\boldsymbol{x}_{i 0}\left(s_{i}, t\right)=\boldsymbol{x}_{i}\left(s_{i}, z_{i}=0, t\right)$ with $s_{i}$ the Lagrangian curvilinear coordinate and $z_{i}$ the spanwise position. The local orientation of the centreline with respect to the incoming flow is $\theta_{i}\left(s_{i}, t\right)$ and $\left(\boldsymbol{e}_{\tau_{i}}, \boldsymbol{e}_{n_{i}}\right)$ denote the local tangent and normal unit vectors to the flag's surface (figure $1 b$ ). An Euler-Bernoulli beam model is used for each flag. Using $L, L / U_{\infty}, \rho H L^{2}$ as characteristic length, time and mass, the non-dimensional equations of motion for $\mathscr{F}_{i}$ read

$$
\frac{\partial^{2} \boldsymbol{x}_{i 0}}{\partial t^{2}}=\frac{\partial}{\partial s_{i}}\left[f_{T_{i}} \boldsymbol{e}_{\tau_{i}}-\frac{1}{U^{* 2}} \frac{\partial^{2} \theta_{i}}{\partial s_{i}{ }^{2}} \boldsymbol{e}_{n_{i}}\right]+\boldsymbol{f}_{\text {fluid }_{i}},
$$

where $f_{T_{i}}\left(s_{i}, t\right)$ is the local tension, acting as a Lagrange multiplier to enforce each flag's inextensibility, namely

$$
\boldsymbol{e}_{\tau_{i}}=\frac{\partial \boldsymbol{x}_{i 0}}{\partial s_{i}}
$$

and $\boldsymbol{f}_{\text {fluid }}\left(s_{i}, t\right)$ is the local fluid force applied on $\mathscr{F}_{i}$. The flags' coupling is purely hydrodynamic, and is therefore included in $\boldsymbol{f}_{\text {fluid }_{i}}$ which depends on the flags' kinematics, and is discussed in detail in the following.

\section{Fluid modelling}

\subsection{Preliminary discussion}

In potential flow, the local pressure force on the flag is directly related to the local flow velocity which can be reconstructed using the Biot-Savart law from the distribution of bound and free vorticity associated with $\mathscr{F}_{i}$ and $\mathscr{W}_{i}$. The relative magnitude of different hydrodynamic contributions can therefore be assessed by considering that of the induced flow velocity. In the case of two slender flags, we look for the dominant hydrodynamic terms depending upon $d$. This first approach provides a rough classification between the contributions of the neighbouring flag and that of the wakes. Further quantitative justifications will be given in subsequent sections. From the Biot-Savart law, the contribution of the velocity field created on $\mathscr{F}_{i}$ by $\mathscr{F}_{j}$ is typically $O\left(d^{-2}\right)$ while, away from the direct neighbourhood of the trailing edge, the wakes contribute as $O\left(L^{-2}\right)$ for $\mathscr{W}_{i}$ (own wake) and $O\left(\left(d^{2}+L^{2}\right)^{-1}\right)$ for $\mathscr{W}_{j}$ (wake of the neighbour). Three cases must therefore be considered. 
(i) If $d \gg L$, the effect of $\mathscr{W}_{i}$ on $\mathscr{F}_{i}$ is dominant over the hydrodynamic coupling between the flags.

(ii) If $d=O(L)$, all the contributions are of the same order and should all be retained.

(iii) If $d \ll L$, the effects of the wakes are negligible compared to hydrodynamic coupling. More precisely a flag region of $O(H)$ near the trailing edge may feel a significant effect of the wakes. It will however be evidenced in the following that the global effect on the flapping dynamics nevertheless remains negligible in this limit. Note that this limitation is intrinsic to the original LAEBT and it is therefore consistent to try to extend this approach to the two-flag case in the range $d \ll L$.

These first considerations indicate that it may be relevant to neglect wakes in the limit of flags in close proximity. In the following, we focus on the range $H \ll d \ll L$ for which the spirit of Lighthill's LAEBT can be extended naturally to account for hydrodynamic coupling: in that range, the effect of the wakes including their complex dynamics appears to remain negligible in front of the hydrodynamic coupling contribution.

\subsection{Methodology}

For a single flag, an asymptotic expansion of the potential flow problem in the limit of small aspect ratio $H^{*}$ (but large displacement) provides the LAEBT formulation of a reactive force that depends exclusively on the local relative velocity of the flag to the background uniform flow (Candelier et al. 2011). This is particularly convenient as a detailed knowledge of the flow around the flag is unnecessary. Note also that this formalism reveals that the non-local effect of the wake is negligible in the slender body limit, and comes at higher order in the expansion in powers of $H^{*}$, as shown by Eloy et al. (2010) in the linear case (EBT). For a freely flapping body, the reactive force obtained here through LAEBT must be complemented by a local resistive force to account for lateral flow separation (Eloy, Kofman \& Schouveiler 2012; Singh, Michelin \& de Langre 2012a).

This local formulation is however lost for two flags, and the hydrodynamic perturbations induced by the second flag must be computed to determine $\boldsymbol{f}_{\text {fuid }_{i}}$. More specifically, in the limit of $H^{*} \ll d^{*}$, these flow perturbations remain subdominant in front of each flag's dominant self-contribution (at least while the flags amplitudes remain small) and vary slowly along the flag's width. The approach followed here is therefore to consider the motion of each flag within the weakly non-uniform local flow field created at its surface by its neighbour's motion. Two steps must be combined, namely (i) the reconstruction of the flow field created by a flapping flag and (ii) a generalization of LAEBT (and of the resistive force) to account for non-uniformities and unsteadiness in the resulting local flow. These two points are detailed below and further combined to propose an extension of the LAEBT approach in the case of two slender flags.

\subsection{Flow created by a flapping flag in the LAEBT}

In this section, an explicit expression of the flow created by a single flapping flag in the large-amplitude regime is obtained. In potential flows, the velocity potential $\phi_{i}$ satisfies Laplace's equation in the fluid domain, that is $\Delta \phi_{i}=0$. In this framework, Green's second identity (see Jackson 1999, for instance) leads to an expression of the velocity potential in the entire fluid domain from the knowledge of the velocity 
potential and its normal derivative (i.e. the normal flow velocity) on the flag and its wake. As a consequence, the flow created in $\boldsymbol{x}$ by the $i$ th flag and its wake reads

$$
\phi_{i}(\boldsymbol{x}, t)=\oint_{\mathscr{F}_{i}+\mathscr{W}_{i}}\left[G\left(\left|\boldsymbol{x}-\boldsymbol{x}_{i}\right|\right) \frac{\partial \phi_{i}\left(\boldsymbol{x}_{i}\right)}{\partial n_{i}}-\phi_{i}\left(\boldsymbol{x}_{i}\right) \frac{\partial G\left(\left|\boldsymbol{x}-\boldsymbol{x}_{i}\right|\right)}{\partial n_{i}}\right] \mathrm{d} S_{i}\left(\boldsymbol{x}_{i}\right),
$$

with $G(r)=-1 /(4 \pi r)$ the free-space Green function of the Laplace equation and where integration should be performed here on both sides of the flag and wake.

In addition, the structure acts as an impermeable surface, so that the normal flow velocity matches that of the flag. For infinitely thin structures, this leads to the continuity of the normal derivative of the flow potential, $\partial \phi\left(\boldsymbol{x}_{i}^{+}\right) / \partial n_{i}-\partial \phi\left(\boldsymbol{x}_{i}^{-}\right) / \partial n_{i}=0$, and the single-layer potential term in (3.1) vanishes

$$
\phi_{i}(\boldsymbol{x}, t)=-\int_{\mathscr{F}_{i}+\mathscr{W}_{i}}\left[\phi_{i}\right]\left(\boldsymbol{x}_{i}\right) \frac{\partial G\left(\left|\boldsymbol{x}-\boldsymbol{x}_{i}\right|\right)}{\partial n_{i}} \mathrm{~d} S_{i}\left(\boldsymbol{x}_{i}\right)
$$

where $\left[\phi_{i}\right]=\phi_{i}\left(\boldsymbol{x}_{i}^{+}\right)-\phi_{i}\left(\boldsymbol{x}_{i}^{-}\right)$corresponds to the velocity potential jump across the flag and wake. In this formalism, the wake is assumed to consist of an infinitely thin vortical sheet of height $H$ extending to infinity. Physically, (3.2) corresponds to the flow induced by bound and free vorticity present in the vicinity of the flag and its wake respectively.

In the slender body limit $(H \ll L)$, the potential jump is given by Candelier et al. (2011) and reads

$$
\left[\phi_{i}\right]\left(\boldsymbol{x}_{i}\right)=-2 \tilde{u}_{n_{i}} \sqrt{H^{*^{2}} / 4-z_{i}^{2}},
$$

with $\tilde{u}_{n_{i}}=\left[\partial \boldsymbol{x}_{i 0} / \partial t-\boldsymbol{e}_{x}\right] \cdot \boldsymbol{e}_{n_{i}}$. Note that this elliptic form of the potential jump is analogous to the small-displacement limit (Lighthill 1970, EBT); effectively, the large-amplitude case can locally be seen as a straight plate having normal relative velocity $\tilde{u}_{n_{i}}$.

Using (3.3), an explicit form of the flow created in the midplane, $z=0$, can be obtained from (3.2) by integrating the contributions of the spanwise direction (for $z_{i}$ from $-H^{*} / 2$ to $\left.H^{*} / 2\right)$ :

$$
\phi_{i}(\boldsymbol{x}, t)=-\int_{0}^{1} \frac{\tilde{u}_{n_{i}}\left(\boldsymbol{x}-\boldsymbol{x}_{i}\right) \cdot \boldsymbol{e}_{n_{i}}}{\pi\left[\left(x-x_{i}\right)^{2}+\left(y-y_{i}\right)^{2}\right]^{1 / 2}}[E(X)-K(X)] \mathrm{d} s_{i}\left(\boldsymbol{x}_{i}\right)+\phi_{W_{i}}\left(\boldsymbol{x}, t, L_{w}\right),
$$

where $X=-H^{* 2} /\left(4\left[\left(x-x_{i}\right)^{2}+\left(y-y_{i}\right)^{2}\right]\right)$ and $K, E$ correspond to the complete elliptic integral functions of the first and second kind respectively (see Abramowitz \& Stegun 1964, p. 590). The first term in (3.4) is the flag's contribution and $\phi_{\mathscr{W}_{i}}$ is the contribution of the wake of non-dimensional size $L_{w}$. Equation (3.4) will allow us to study the influence of the wake on the flow reconstruction in the following paragraph by means of a simple wake model. Later on, an additional assumption termed the far-field approximation will be introduced for convenience and its range of validity will also be examined. 
(a)

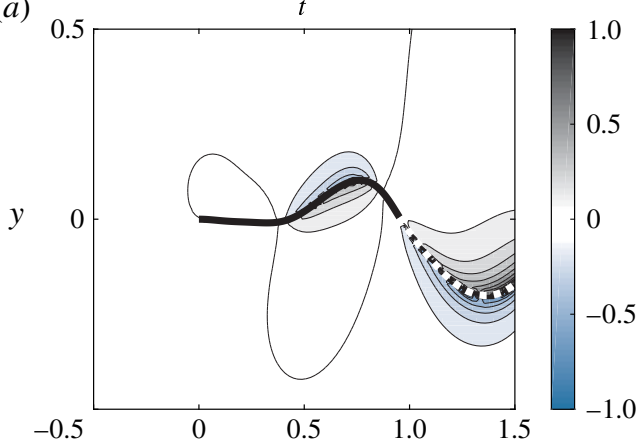

(c)

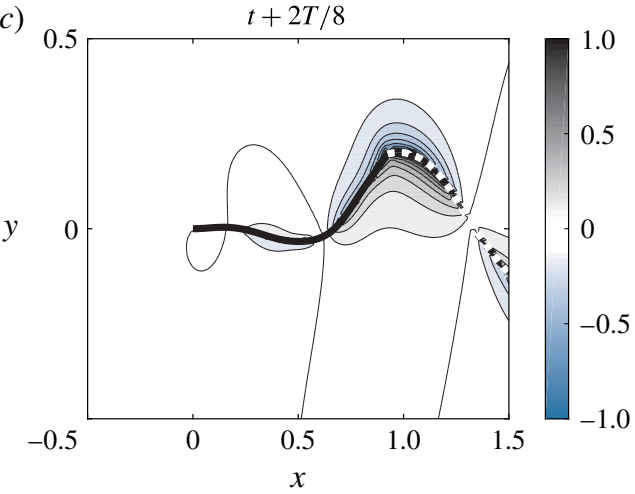

(b)

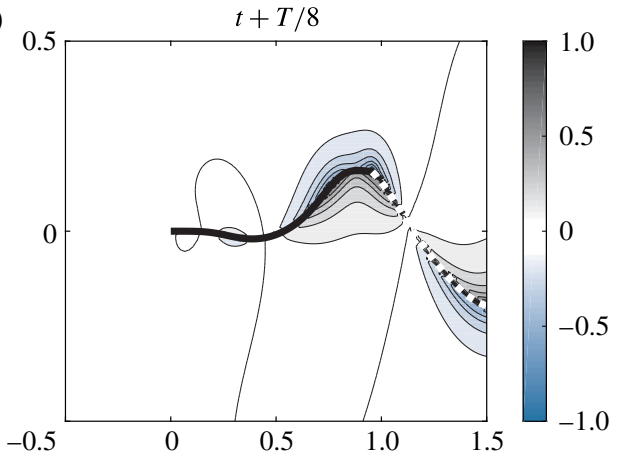

(d)

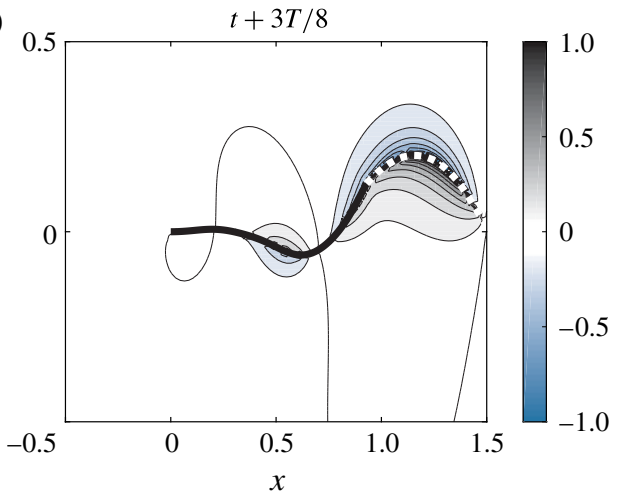

FIGURE 2. (Colour online) Flow created in the midplane $z=0$ during a flapping sequence for $M^{*}=10, U^{*}=20$ and $H^{*}=0.1$. Instantaneous normalized velocity potential obtained from (3.2) and (3.3) using a frozen wake model of non-dimensional length $L_{w}=2$. The flag's (respectively wake's) position is shown by a thick black (respectively dashed) line.

\subsubsection{Role of the wake}

Solving (3.4), the flow around the flag is obtained everywhere provided the wake characteristics (position and circulation) are known. In order to assess the effect of the wake on the created flow, a simplified wake model is constructed. We neglect auto-induction and therefore assume that the vorticity shed at the trailing edge is only advected downstream by the uniform flow. This wake model is referred to as frozen wake in the following and has already been considered in previous studies on flexible bodies (see Candelier et al. 2011, for instance). Figure 2 shows an example of velocity potential contours obtained from (3.4) with such a simplified wake model. From this flapping sequence, it can be seen that the flow varies in the streamwise direction on length scales of the order of the flapping wavelength, that is of the order of $L$ for the first flapping modes.

The effect of the wake on the created flow field is now investigated in figure $3(a)$ which corresponds to the relative error map obtained by comparison between results with a wake of non-dimensional length $L_{w}=2$ and results obtained without taking the wake into account. The white area corresponds to locations where the error associated with ignoring the wake is less than $1 \%$ while the darkest blue region indicates an error larger than $30 \%$. For this value of $H^{*}$, the length of the wake does not significantly change the result provided $L_{w} \geqslant 1$ (not shown). In these cases, we therefore obtain 

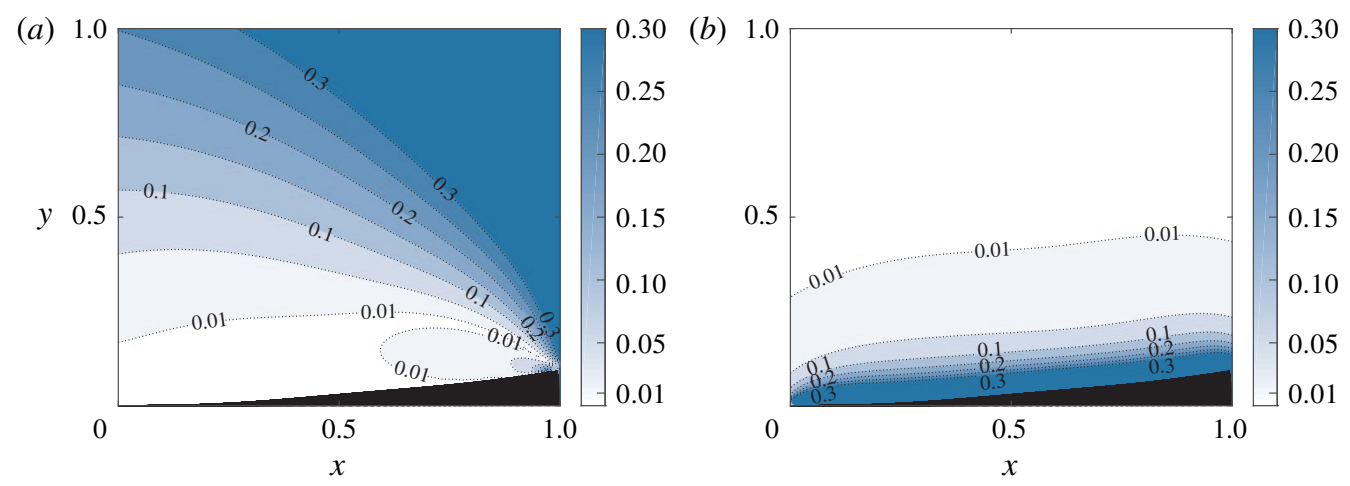

FIGURE 3. (Colour online) Relative error maps for the flow reconstruction in the midplane $z=0$ in the case $M^{*}=3, U^{*}=15$ and $H^{*}=0.1$. (a) Error obtained when the wake is neglected. The error is defined as $\mid\left[\right.$ r.m.s. $\left(v_{L_{w}=2}\right)-$ r.m.s. $\left.\left(v_{L_{w}=0}\right)\right] /$ r.m.s. $\left(v_{L_{w}=2}\right) \mid$ where the case without wake is compared to the case with a frozen wake of non-dimensional length $L_{w}=2$. (b) Error due to far-field approximation $(F F)$ defined as $\mid\left[\right.$ r.m.s. $\left(v_{F F}\right)-$ r.m.s. $(v)] /$ r.m.s. $\left(v_{F F}\right) \mid$. Wakes are neglected in this case. The black area delineates the envelope of the flapping flag.

that the wake influence is weak while $y<0.3$ (for $H^{*}=0.1$ ) and far enough from the trailing edge as the error becomes important for distances $O\left(H^{*}\right)$ from this location. When considering two flags side by side, it is reasonable to neglect wake effects in the coupling terms within the range $d^{*}<0.3$. Even though the flow is not well predicted close to the trailing edge, it will be verified that it does not significantly affect the global dynamics whose prediction is the main focus of the present article.

\subsubsection{Far-field approximation $(F F)$}

Equation (3.4) can be reformulated when looking at the flow field at distances from the flag that are large compared to $H^{*}$. Using the asymptotic expansion of $E(X)$ and $K(X)$ for $X \ll 1$, the following approximation of the flow potential is obtained in the midplane $z=0$,

$$
\phi_{i}(\boldsymbol{x}, t)=-\frac{H^{* 2}}{16} \int_{0}^{1} \frac{\tilde{u}_{n_{i}}\left(\boldsymbol{x}-\boldsymbol{x}_{i}\right) \cdot \boldsymbol{e}_{n_{i}}}{\left[\left(x-x_{i}\right)^{2}+\left(y-y_{i}\right)^{2}\right]^{3 / 2}} \mathrm{~d} s_{j}\left(\boldsymbol{x}_{i}\right),
$$

if $\phi_{W_{i}}$ is further neglected. This approximation is equivalent to assuming that the Green function is independent of the spanwise coordinate in (3.2). The range of validity of the far-field approximation can be estimated from figure $3(b)$ where the error shows that this approximation essentially affects the flow for lateral distances to the flag of order $H^{*}$. Equation (3.5) therefore provides a simplified version of (3.4) which is valid far from the flag (compared to $H^{*}$ ) and in regions where wakes do not contribute significantly to the flow; it is therefore valid for intermediate lateral distances $\left(0.1<y<0.3\right.$ in the present case where $\left.H^{*}=0.1\right)$. This form of the velocity potential given by (3.4) is introduced here only as a matter of convenience as it allows for faster simulations and simplifies the analysis; but the present method could be applied directly using (3.4). In addition, as detailed in the following, far-field approximation is consistent with further modelling steps for two flags and has little impact on the global dynamic when $d^{*}>H^{*}$. 


\subsection{LAEBT in a weakly non-uniform potential flow}

Lighthill's LAEBT was recently generalized by Candelier et al. (2013) to the case of a slender structure in a weakly non-uniform potential flow. In the classical LAEBT, the uniform incoming flow and the flow created by the flapping flag can respectively be termed ambient flow and perturbed flow. Candelier et al. (2013) extends LAEBT for weakly non-uniform ambient flows, i.e. for cases where the ambient flow varies on length scales much larger than the cross-section dimensions.

Under this assumption, the local ambient flow can be expended in Taylor series in each cross-section around the centreline of the body. This provides a decomposition of the perturbed flow which accounts for non-uniformities of the ambient flow. Such a procedure eventually leads to an expression for the fluid force exerted on a body immersed in a weakly non-uniform and potential flow, which has been successfully implemented to simulate swimming of a slender fish in a von Kármán vortex street (Candelier et al. 2013).

In this paragraph, we present a brief summary of this result, and use dimensional quantities (in capital letters) to clarify the physical origin of the different contributions to the force. The reader is referred to the original study of Candelier et al. (2013) for more details. Considering a slender structure with centreline position $\boldsymbol{X}_{0}$ immersed in a potential and weakly non-uniform ambient flow with velocity $\boldsymbol{V}(\boldsymbol{X}, T)$ and pressure $P(X, T)$, body-fitted coordinates $X^{n}$ and $X^{\tau}$ are introduced and respectively correspond to normal and tangential positions. For planar motions of the structure, the dimensional local pressure force exerted on the solid is obtained as (see Candelier et al. 2013, equation (4.5))

$$
\boldsymbol{F}=-\left.S \nabla P\right|_{X=X_{0}}-\left[\frac{\partial M_{a} U_{n} \boldsymbol{e}_{n}}{\partial T}-\frac{\partial M_{a} U_{n} U_{\tau} \boldsymbol{e}_{n}}{\partial X^{\tau}}+\frac{1}{2} \frac{\partial M_{a} U_{n}^{2} \boldsymbol{e}_{\tau}}{\partial X^{\tau}}\right]-\left.M_{a} U_{n} \frac{\partial \boldsymbol{V}}{\partial X^{n}}\right|_{\boldsymbol{X}=\boldsymbol{X}_{0}},
$$

with $S$ the surface area of the body's local cross-section and $M_{a}$ the added mass associated with its normal displacement. Note that the body shape (and therefore $S$ and $M_{a}$ ) may slowly vary along the tangential direction in this formalism. In addition, $U_{n}$ and $U_{\tau}$ correspond to the components of the local relative velocity between the solid and the ambient flow which are defined as

$$
U_{n} \boldsymbol{e}_{n}+U_{\tau} \boldsymbol{e}_{\tau}=\frac{\partial \boldsymbol{X}_{0}}{\partial T}-\boldsymbol{V}\left(\boldsymbol{X}=\boldsymbol{X}_{0}\right)
$$

The physical origin of the three terms in (3.6) can be understood as follows.

(i) The first term is due to non-uniformities of the ambient pressure and can physically be interpreted as a generalization of Archimedes' force which vanishes in the case of the infinitely thin flag considered here $(S \rightarrow 0)$.

(ii) The second term corresponds to the classical LAEBT expression (see Singh, Michelin \& de Langre $2012 b$, for instance) in which the relative velocity defined by (3.7) now takes into account the non-uniformities of the ambient flow.

(iii) The third term is an additional contribution due to structure's motion within the ambient velocity gradient.

\subsection{LAEBT in a weakly non-uniform potential flow: application to flag geometry}

Moving back to the non-dimensional framework introduced in $\S 2$ the above theory is now applied to the specific flag geometry investigated in this work. Considering 
a flag $\mathscr{F}_{i}$ placed in a weakly non-uniform and potential ambient flow $\boldsymbol{v}$, the general expression of the reactive fluid force shown in (3.6) simplifies for an infinitely thin flag with uniform added mass coefficient $\left(m_{a}=\pi / 4\right)$. Furthermore, applying the inextensibility of the structure and irrotationality of the ambient flow, the tangential component of the force in (3.6) can be shown to vanish exactly. If the ambient flow is further symmetric with respect to the midplane, the local reactive force exerted on $\mathscr{F}_{i}$ is purely normal and its non-dimensional form can be written as

$$
\boldsymbol{f}_{\text {react }_{i}}=-m_{a} H^{*} M^{*}\left(\frac{\partial u_{n_{i}}}{\partial t}-\frac{\partial u_{n_{i}} u_{\tau_{i}}}{\partial s_{i}}+\frac{u_{n_{i}}^{2}}{2} \frac{\partial \theta_{i}}{\partial s_{i}}+u_{n_{i}} \boldsymbol{e}_{n_{i}} \cdot\left[\left.\nabla \boldsymbol{v}\right|_{x_{i}=x_{i 0}}\right] \cdot \boldsymbol{e}_{n_{i}}\right) \boldsymbol{e}_{n_{i}}
$$

with relative velocity

$$
u_{n_{i}} \boldsymbol{e}_{n_{i}}+u_{\tau_{i}} \boldsymbol{e}_{\tau_{i}}=\frac{\partial \boldsymbol{x}_{i 0}}{\partial t}-\boldsymbol{v}\left(\boldsymbol{x}_{i}=\boldsymbol{x}_{i 0}\right)
$$

Equation (3.8) generalizes LAEBT to the motion of a flag in non-uniform flow. Inhomogeneities of the ambient flow appear explicitly in the last term and implicitly in the others through the relative velocity defined in (3.9). The EBT can be extended to non-uniform flows in a similar fashion, and corresponds to the leading-order expansion of the previous equation in the limit of small displacements. Since $\boldsymbol{v}$ is only weakly non-uniform, the last term in (3.8) is quadratic in the small flapping amplitude regime and should be discarded: the force expression in the EBT is therefore formally identical in uniform and weakly non-uniform flows and only differ in the definition of the appropriate relative velocity.

Additionally, the reactive contribution given by (3.8) from LAEBT must be complemented by a resistive contribution which should also be modified to account for flow non-uniformities. In line with Eloy et al. (2012), skin drag is neglected and we model the drag associated with lateral flow separation as

$$
\boldsymbol{f}_{\text {resist }}=-\frac{1}{2} M^{*} C_{d} u_{n_{i}}\left|u_{n_{i}}\right| \boldsymbol{e}_{n_{i}},
$$

with $C_{d}=1.8$ for a flat plate and $u_{n_{i}}$ defined in (3.9).

The combination of $\boldsymbol{f}_{\text {react }}$ and $\boldsymbol{f}_{\text {resist }}$ finally provides a model for the local fluid force applied on a slender flag immersed in the weakly non-uniform flow $\boldsymbol{v}$. The weak nonuniformity means that the components of $v$ are not significantly varying over $O\left(H^{*}\right)$ length scales. In particular, this explains why only the flow at the centreline is needed to obtain the fluid forces in (3.8) and (3.10). In the following, these expressions are used to model the configuration of two slender flags by considering that the ambient flow corresponds to the superposition of the uniform axial flow and the flow created by the neighbouring flag.

\subsection{LAEBT for two slender flags}

For a specific intermediate range of non-dimensional distances $d^{*}$, combination of the results of the two previous sections provides an extension of LAEBT to the case of two slender and infinitely thin structures placed side by side. The underlying idea is to consider that each flag is flapping in the non-uniform flow corresponding to the superposition of the uniform incoming flow and the flow created by its neighbour. From $\$ 3.4$ the local fluid forces exerted on each flag can therefore be modelled as $\boldsymbol{f}_{\text {fuid }}$ i $\boldsymbol{f}_{\text {reacti }}+\boldsymbol{f}_{\text {resisti }}$, where $\boldsymbol{f}_{\text {reacti }}$ and $\boldsymbol{f}_{\text {resisti }}$ are given by (3.8) and (3.10) respectively, 
and in which the velocity $\boldsymbol{v}$ must be replaced by $\boldsymbol{e}_{x}+\nabla \phi_{j}$, where $\phi_{j}$ corresponds to the velocity potential created by $\mathscr{F}_{j}$ (with $j \neq i$ ) and is obtained from (3.4).

Based on the conclusions of $\S 3.3$ and unless otherwise stated, the influence of the wakes is neglected in the following which focuses on regimes where the flags are close compared to their length. Doing so effectively overlooks the modification of the flow field in the trailing edge's immediate vicinity, but we show in the following that this assumption has essentially no effect on the overall dynamics.

In addition, the LAEBT extension leading to the reactive force is only valid in the case of a weakly non-uniform flow, i.e. if the components of $\boldsymbol{v}$ are not varying much on length scales of the order of $H^{*}$. The present fluid model is therefore valid if all parts of the flags remain far compared to $H^{*}$, a condition which corresponds to $H^{*} \ll d^{*}$ for small amplitudes. Large-amplitude cases require more care as it depends on the synchronization phase between flags, but it is worth noting that it will automatically hold in the case of in-phase motion for which the distance between flags does not get significantly smaller than $d^{*}$. This weakly non-uniform restriction legitimates the far-field approximation and $\phi_{j}$ is therefore calculated using (3.5).

As a conclusion, (2.2)-(2.3) for both flags' dynamics coupled to the fluid model provided by (3.8)-(3.10) with $\boldsymbol{v}=\boldsymbol{e}_{x}+\nabla \phi_{j}$ and $\phi_{j}$ given by (3.5) provide a model for two side by side flags with geometric parameters in the range $H^{*} \ll d^{*} \ll 1$.

\section{Linear case}

For small lateral displacements $y_{i}\left(s_{i}, t\right)$ of flag $\mathscr{F}_{i}(i=1,2)$, equations (2.2)-(2.3) and (3.8)-(3.10) can be linearized around the equilibrium position, $y_{i}(s, t)=0$, leading to the EBT formulation of the two-flag problem:

$$
\frac{\partial^{2} y_{i}}{\partial t^{2}}+\frac{1}{U^{* 2}} \frac{\partial^{4} y_{i}}{\partial s_{i}^{4}}=-m_{a} H^{*} M^{*}\left(\frac{\partial^{2} y_{i}}{\partial t^{2}}+2 \frac{\partial^{2} y_{i}}{\partial t \partial s_{i}}+\frac{\partial^{2} y_{i}}{\partial s_{i}^{2}}\right)+m_{a} H^{*} M^{*}\left(\frac{\partial}{\partial t}+\frac{\partial}{\partial s_{i}}\right) v_{j i},
$$

with $v_{j i}$ the linear lateral velocity induced by $\mathscr{F}_{j}(j \neq i)$ on $\mathscr{F}_{i}$ obtained from (3.5)

$$
v_{j i}\left(s_{i}\right)=\frac{H^{* 2}}{16} \int_{0}^{1}\left(\frac{\partial y_{j}}{\partial t}+\frac{\partial y_{j}}{\partial s_{j}}\right) \frac{2 d^{* 2}-\left(s_{i}-s_{j}\right)^{2}}{\left[d^{* 2}+\left(s_{i}-s_{j}\right)^{2}\right]^{5 / 2}} \mathrm{~d} s_{j} .
$$

Equations (4.1)-(4.2) provide a simplified linear system for $y_{1}(s, t)$ and $y_{2}(s, t)$, which also provides important insight on the different fluid contributions (right-hand side of (4.1)) and justifies a posteriori some of the modelling assumptions. This discussion will be made using a classification of the fluid terms in powers of $H^{*}$ and $d^{*}$.

The first fluid term of (4.1) corresponds to the traditional EBT contribution (see Eloy, Souilliez \& Schouveiler 2007, for instance), while the second results from the coupling with the neighbouring flag. Both fluid terms correspond to the flow forcing over the local cross-section, leading to a pre-factor $H^{*}$ with the present set of nondimensional numbers. For the coupling term, a spanwise integration is performed on the forcing flag leading to the factor $H^{* 2}$ in the induced velocity. The integral in (4.2) scales as $1 / d^{* 2}$ and the coupling terms therefore finally scales as $O\left(H^{* 3} / d^{* 2}\right)$. We therefore obtain that EBT and coupling terms scale respectively as $O\left(H^{*}\right)$ and $O\left(H^{* 3} / d^{* 2}\right)$. In order to validate the model, these terms should be compared to the first correction of EBT for a single flag (which includes the first-order contribution 


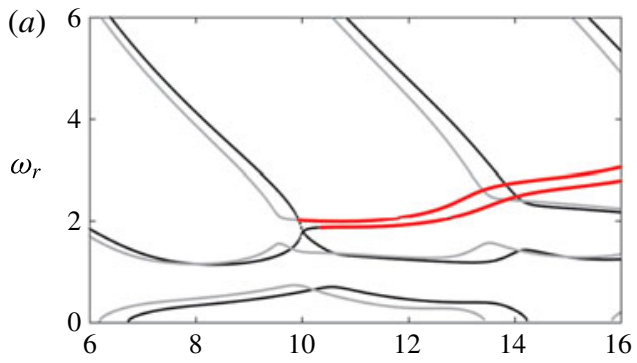

(b)
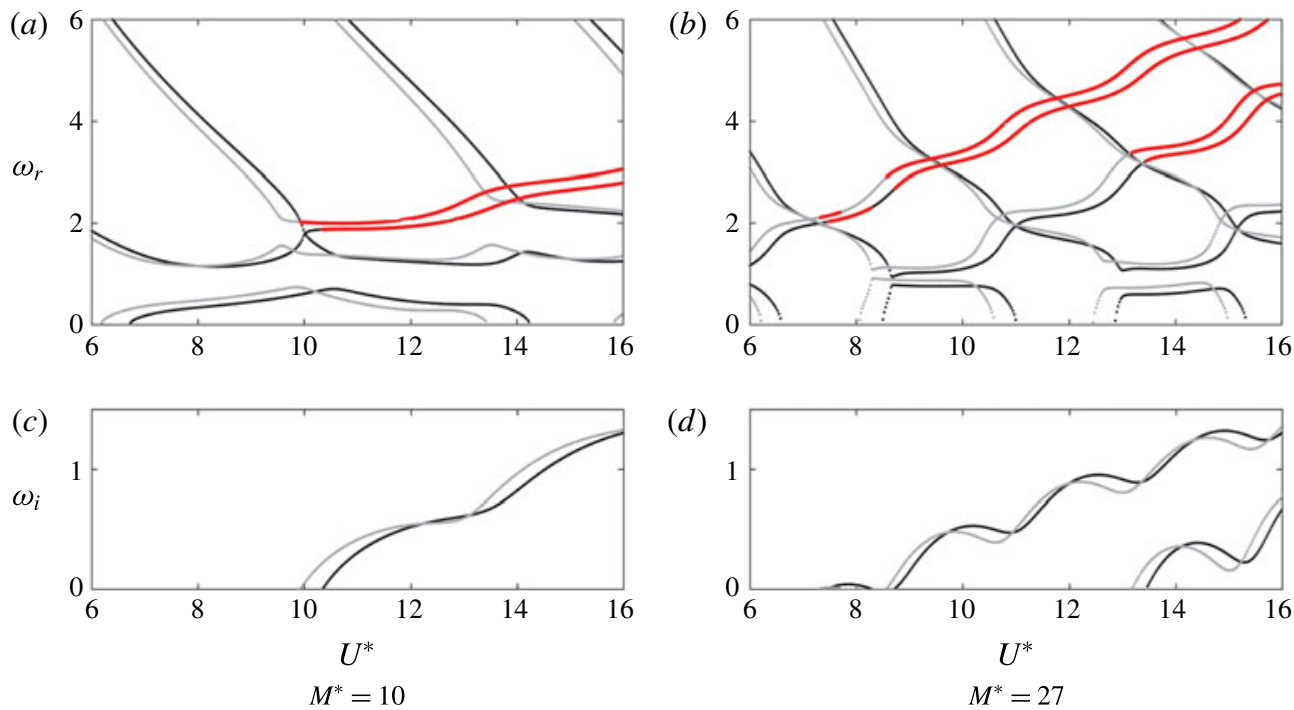

$(d)$

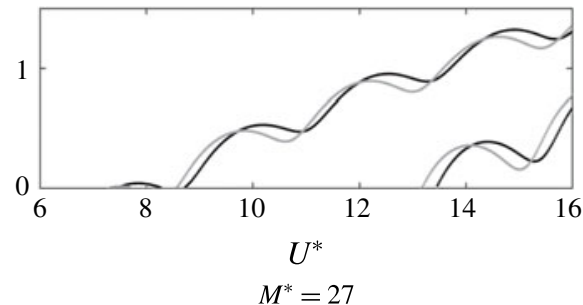

Figure 4. (Colour online) Frequencies $(a, b)$ and growth rates $(c, d)$ for $H^{*}=0.1$ and $d^{*}=0.15$. The black lines (respectively grey lines) correspond to in-phase (respectively out-of-phase) modes. Frequencies corresponding to positive growth rates are shown in bold red.

of the flag's own wake), which scales as $O\left(H^{* 3} \ln H^{*}\right)$ (Eloy et al. 2010). For a flag aspect ratio $H^{*}$, the coupling term decreases with $d^{*}$ and becomes as small as the EBT correction at some point. For $H^{*}=0.1$ for instance, it is found that the coupling term has the same magnitude as the EBT correction for $d^{*} \approx 0.7$. For distances around and larger than this value, it would therefore be inconsistent to retain hydrodynamic coupling terms while neglecting EBT corrections. The present approach therefore only works while $d^{*} \ll 1$. In order to extend this approach for larger values of $d^{*}$, EBT corrections (and therefore the contribution of the flag's own wake) should be included and the coupling terms due to the neighbour's wake should also be taken into account.

In addition, it should be noted that higher-order coupling terms obtained by taking into account, during the flow reconstruction, the flag's immersion in a non-uniform flow (i.e. replacing $\tilde{u}_{n_{i}}$ by $u_{n_{i}}$ in (3.3)) scale as $O\left(H^{* 5} / d^{* 4}\right)$. This term is therefore neglected in the present study.

Searching for $y_{i}\left(s_{i}, t\right)=Y_{i}\left(s_{i}\right) \mathrm{e}^{-\mathrm{i} \omega t}$ with clamped-free boundary conditions and using spatial Chebyshev collocation, the system (4.1)-(4.2) is rewritten as a generalized eigenvalue problem for $\omega$ and $\left[Y_{1}\left(s_{1}\right), Y_{2}\left(s_{2}\right)\right]$. The real and imaginary parts of $\omega$ are the frequency $\omega_{r}$ and growth rate $\omega_{i}$ of the corresponding mode, respectively, and $\omega_{i}>0$ denotes instability. The problem's symmetry imposes that $Y_{1}= \pm Y_{2}$, i.e. flags are either in-phase or out-of-phase (Michelin \& Llewellyn Smith 2009).

Figure 4 shows that the two-flag configuration remains stable up to a critical reduced velocity $U_{c}^{*}$ where the out-of-phase mode becomes unstable for the values of $M^{*}, H^{*}$ and $d^{*}$ considered. Increasing $U^{*}$ further, successive switches are observed in the synchronization of the most unstable mode (out-of-phase/in-phase). The map of the most unstable mode in the parameter space $\left(M^{*}, U^{*}\right)$ is shown on figure 5 and reveals that such switching phenomena are found for large values of $M^{*}$. From this general picture, we conclude that in-phase modes are predominantly expected 


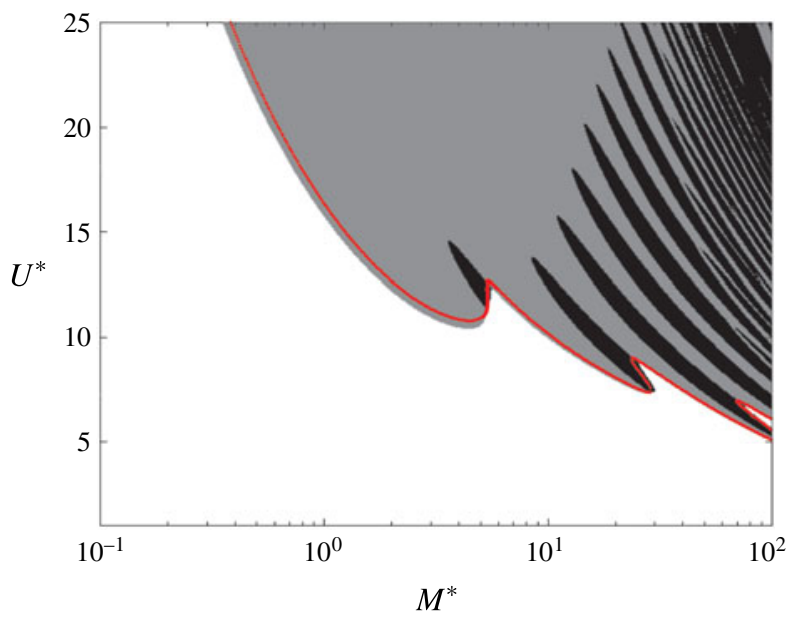

FIgURE 5. (Colour online) Synchronization in the most unstable mode for $H^{*}=0.1$ and $d^{*}=0.15$. In-phase (respectively out-of-phase) dominant modes correspond to black (respectively grey) regions. White regions correspond to a stable configuration. The red line indicates the stability threshold of a single flag $(d \rightarrow \infty)$.

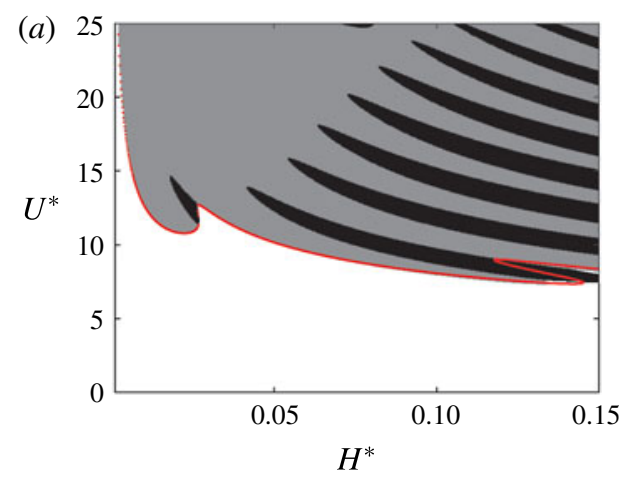

$d^{*}=0.15, M^{*}=20$ (b)

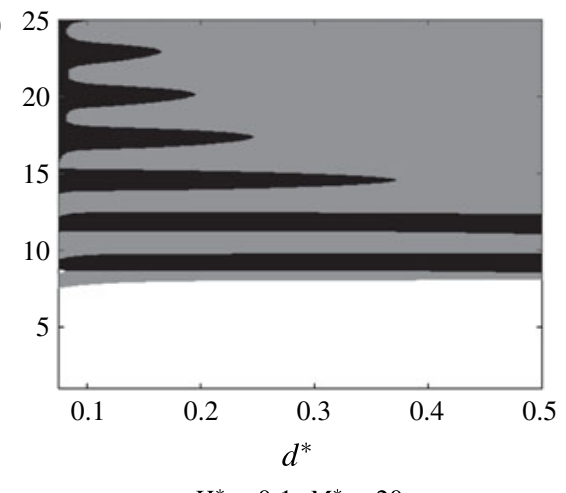

$H^{*}=0.1, M^{*}=20$

FiguRE 6. (Colour online) Effect of $H^{*}(a)$ and $d^{*}(b)$ on the synchronization in the most unstable mode. Colour conventions are identical to those in figure 5.

for large $M^{*}$ and out-of-phase modes for small $M^{*}$, a trend reminiscent of the linear predictions in the two-dimensional limit (Michelin \& Llewellyn Smith 2009). In addition, the presence of a neighbour has a destabilizing effect for most $M^{*}$ as evidenced on figure 5 by comparison with the results for a single flag.

Figure 6(a) reveals that out-of-phase modes are predominant when $H^{*}$ tends to zero while both modes are found for $H^{*}$ comparable to $d^{*}$. In addition, comparison with the single-flag threshold shows that the effect of the neighbour becomes small when $H^{*} \ll 1$, which is consistent with (4.2) and the $O\left(H^{* 2}\right)$ of the induced velocity scales. Figure $6(b)$ reveals that in-phase modes are obtained for small values of $d^{*}$ while outof-phase modes dominate at larger distances. Noticeably, this latter trend also agrees with two-dimensional experiments and numerical results (Zhang et al. 2000; Zhu \& Peskin 2003). 

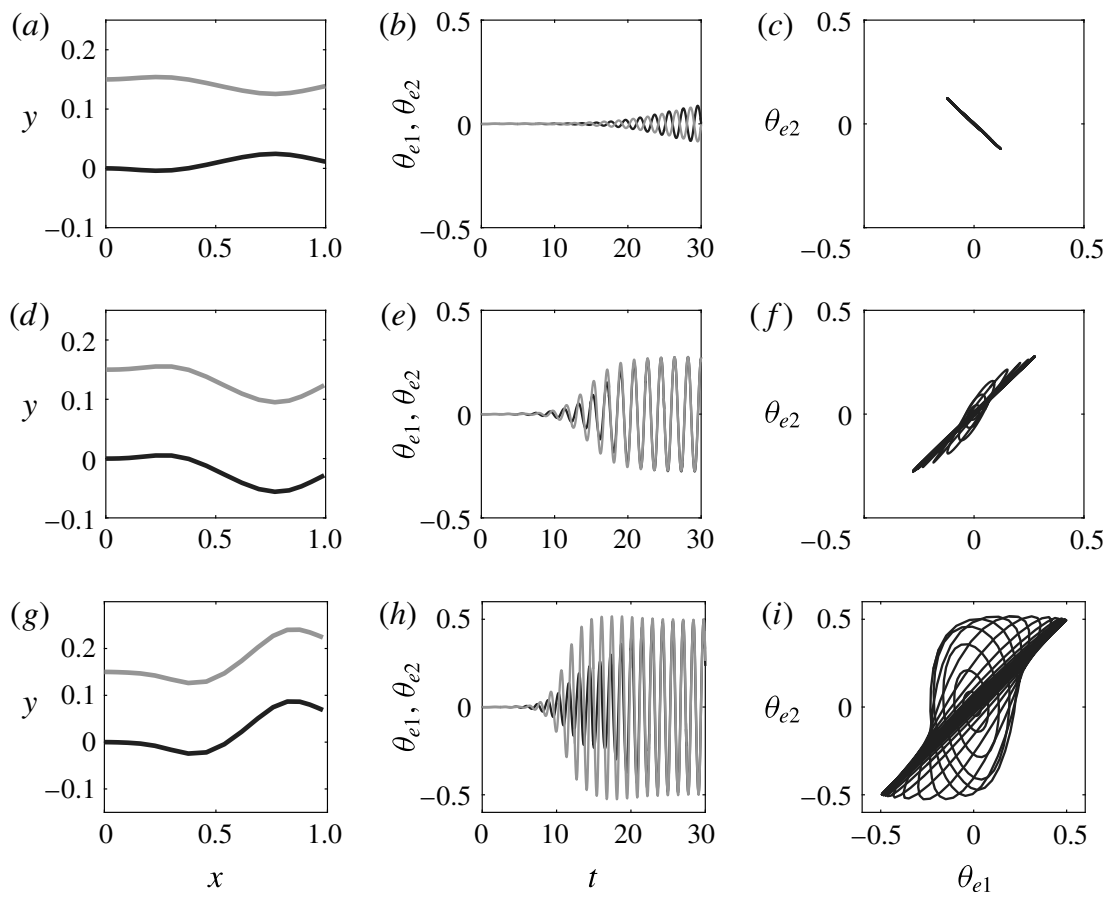

FIGURE 7. Flapping dynamics for $H^{*}=0.1, d^{*}=0.15, M^{*}=27$ and $U^{*}=9(a-c), U^{*}=10$ $(d-f)$ and $U^{*}=11.7(g-i) .(a, d, g)$ Show the flags' instantaneous position. $(b, e, h)$ and $(c, f, i)$ Show the evolution of trailing edge angles $\theta_{e i}$.

\section{Large-amplitude case}

The numerical approach presented in more details in Michelin \& Doaré (2013) is extended here to solve the nonlinear coupled equations for the dynamics of the two flags, (2.2)-(2.3), (3.5) and (3.8)-(3.10). The beam dynamics is projected along the normal and tangential directions, and the projections on $\boldsymbol{e}_{\tau_{i}}$ provide the tensions $f_{T_{i}}$ in both flags which are substituted into the dynamics along the normal directions. Using a semi-implicit time-stepping scheme, the entire system then becomes a nonlinear equation for $\left[\partial \theta_{1} / \partial s_{1}, \partial \theta_{2} / \partial s_{2}\right]$ solved iteratively at each time step using Broyden's method. Eventually, flapping characteristics can be reconstructed from curvature distributions using clamped-free boundary conditions. Initially, one flag is straight and a small uniform curvature is imposed on the second flag. This ensures that initial conditions do not promote in-phase or out-of-phase dynamics.

Figure 7 illustrates the resulting dynamics: the small perturbations grow on the perturbed flag and set the second flag into motion, leading to exponential growth and saturation of both flapping amplitudes. After a transient regime, flags settle in a permanent regime, either in-phase or out-of-phase, with the same flapping amplitude and frequency. For larger $d^{*}$ (not shown), a similar behaviour is found and the time necessary to reach the saturation of the flapping amplitude is roughly independent of $d^{*}$. However, the time required to reach synchronization (i.e. the time necessary for the relative phase to converge to its long-term value) increases significantly with $d^{*}$ : flags are not necessarily in-phase or out-of-phase when their flapping amplitude saturates, and a long transient regime may be required before reaching synchronization. Saturation and synchronization therefore occur on different 


\begin{tabular}{llccc} 
& Configuration & Frequency & Amplitude & Synchronization \\
$U^{*}=9$ & $L_{w}=0$, FF approx. & 3.27 & 0.0340 & out-of-phase \\
& $L_{w}=1$, FF approx. & 3.24 & 0.0354 & out-of-phase \\
& $L_{w}=0$ & 3.26 & 0.0336 & out-of-phase \\
& $L_{w}=1$ & 3.24 & 0.0349 & out-of-phase \\
& $L_{w}=2$ & 3.24 & 0.0348 & out-of-phase \\
& LAEBT single flag & 3.14 & 0.0271 & - \\
\hline$U^{*}=10$ & $L_{w}=0$, FF approx. & 3.42 & 0.0766 & in-phase \\
& $L_{w}=1$, FF approx. & 3.44 & 0.0774 & in-phase \\
& $L_{w}=0$ & 3.43 & 0.0761 & in-phase \\
& $L_{w}=1$ & 3.44 & 0.0769 & in-phase \\
& $L_{w}=2$ & 3.44 & 0.0769 & in-phase \\
& LAEBT single flag & 3.49 & 0.0685 & - \\
\hline$U^{*}=11.7$ & $L_{w}=0$, FF approx. & 4.56 & 0.1078 & in-phase \\
& $L_{w}=1$, FF approx. & 4.57 & 0.1076 & in-phase \\
$L_{w}=0$ & 4.57 & 0.1079 & in-phase \\
$L_{w}=1$ & 4.58 & 0.1077 & in-phase \\
$L_{w}=2$ & 4.58 & 0.1078 & in-phase \\
LAEBT single flag & 4.68 & 0.1065 & -
\end{tabular}

TABLE 1. Influence of the model hypotheses on the flapping characteristics for $H^{*}=0.1$, $d^{*}=0.15$ and $M^{*}=27$. For each $U^{*}$, the first line corresponds to cases shown on figure 7 .

time scales. This is consistent with saturation being mainly driven by the flag's own dynamics and synchronization resulting from hydrodynamic coupling: the latter become weaker when $d^{*}$ is increased. It should be noted nevertheless that the flapping amplitude $A$ (defined as the half of the peak-to-peak amplitude corresponding to the trailing edge's lateral position in the saturated regime) may be slightly modified during the synchronization process (table 1).

The flapping characteristics (i.e. the amplitude, frequency and synchronization type) associated with the dynamics presented in figure 7 are reported in table 1 (bold), which validates the different approximations made (i.e. neglecting the wake and the far-field approximation). In addition, the single flag case is also reported for comparison. Results show that both wakes and far-field approximation have little influence on the flapping characteristics for the cases considered here. In particular, the influence of both approximations remain small in front of that of hydrodynamic coupling which is estimated through comparison with the single-flag case. This validates the present model for the physical parameters considered in table 1 .

For the same set of parameters, figures 8 and 9 compare large-amplitude results with the linear predictions. Figure 8 displays the linear growth and the saturated amplitude obtained from nonlinear simulations. As can be seen, final states obtained from LAEBT simulations roughly correspond to the most unstable mode in the linear predictions. The apparent discrepancy found at the lowest threshold $\left(U^{*} \approx 7.5\right)$ is due to the very small amplification rate which requires long simulation times to obtain the final saturated state. It has been checked that the out-of-phase area predicted close to threshold in the linear case eventually emerges from LAEBT simulations (but with a very weak amplitude) for very long simulation times. Thresholds are therefore well reproduced. Figure 9 compares the linear frequencies already presented in figure 4 to the flapping frequencies of the final state obtained from the large-amplitude 


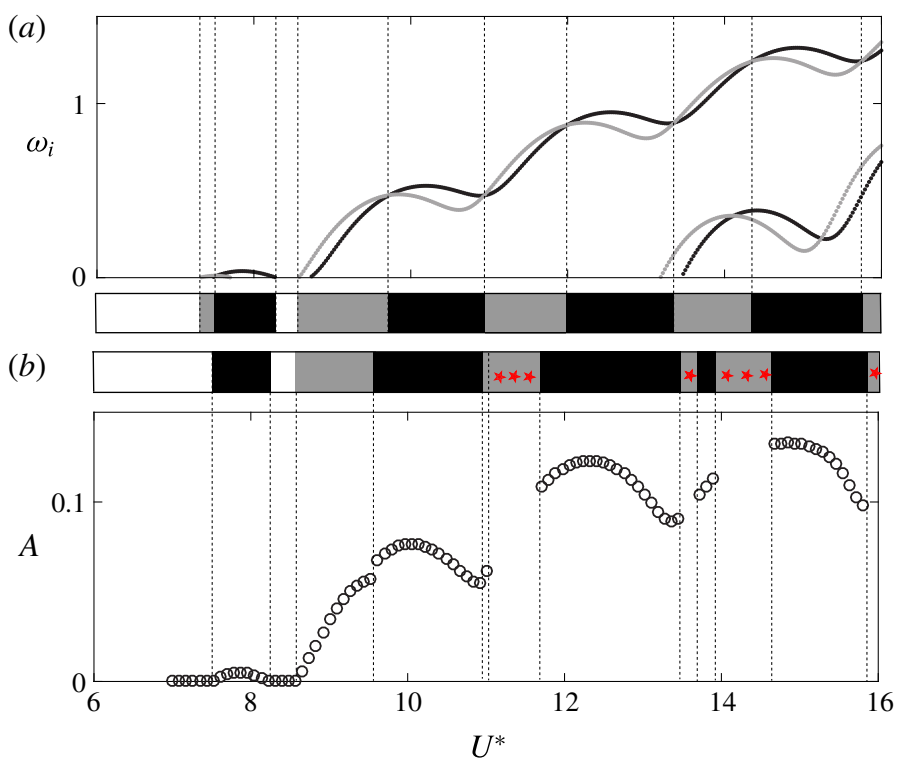

Figure 8. (Colour online) Comparison of linear and nonlinear results for $H^{*}=0.1, d^{*}=$ 0.15 and $M^{*}=27$. (a) Linear growth rates of in-phase (black) and out-of-phase modes (grey). (b) Saturated amplitude obtained from nonlinear simulations. In both cases, the associated coloured stripes indicate the flags' synchronization in the most unstable mode and saturated regime: in-phase (black) or out-of-phase (grey). White regions correspond to a stable assembly. Red stars correspond to cases where simulations are stopped due to touching flags.

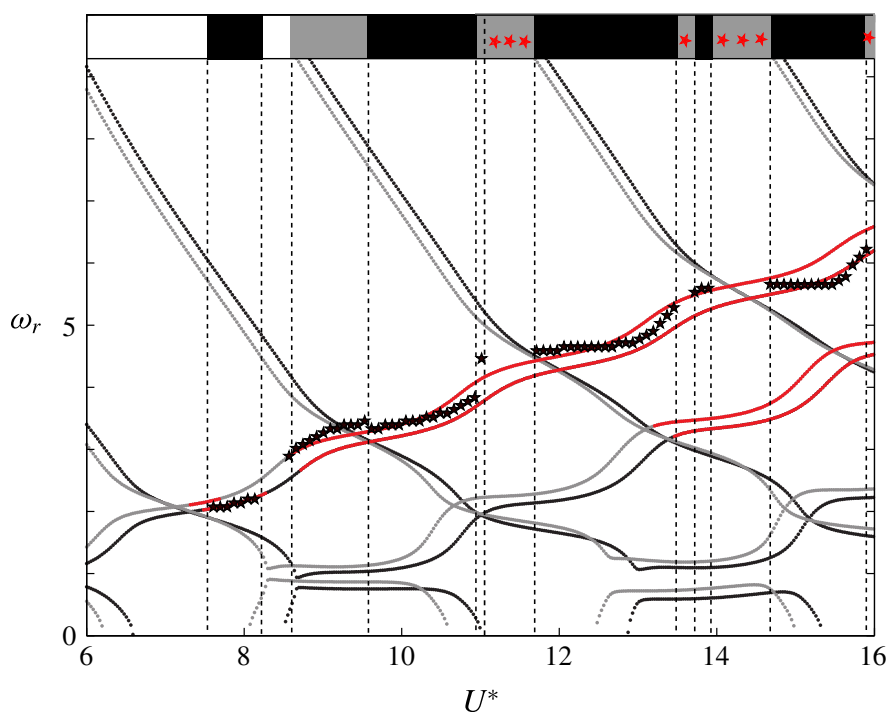

FIGURE 9. (Colour online) Comparison of linear and nonlinear frequencies. Linear results correspond to figure $4(b)$, nonlinear frequencies in the final state are represented in black stars. Parameters and colour conventions are identical to those in figure 8. 


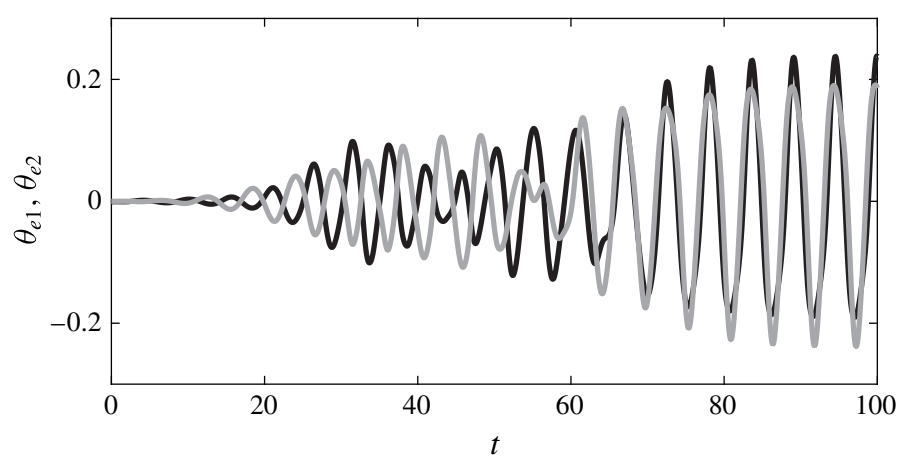

FIGURE 10. Trailing edge angles as a function of time from LAEBT simulation for $M^{*}=3, d^{*}=0.1$ and $U^{*}=15$.

simulations. A good agreement is found and large-amplitude results confirm that the out-of-phase dynamics is associated with larger frequencies than in-phase dynamics; a trend also reported in the two-dimensional experiments by Zhang et al. (2000).

However, figure 8 shows that the nonlinear dynamics may differ from linear predictions regarding the flags' synchronization based on the most unstable eigenmodes (e.g. for $U^{*} \approx 11.7$ ). This behaviour corresponds to figure 7 (bottom line) where the system is dominated by an out-of-phase mode at the beginning of the transient stage (the out-of-phase mode is most unstable) before nonlinearities become important and switch the system to in-phase synchronization. This phenomenon is highlighted for another set of parameters in figure 10 where the growth of the linear out-of-phase prediction is clearly seen before the system eventually settled in-phase. In both cases, the nonlinearities therefore appear to favour in-phase synchronization. This type of nonlinear selection is only observed when the most unstable in-phase and out-of-phase modes have similar growth rates, and can be seen as a competition between two modes which is expected to occur frequently for large values of $M^{*}$ (see figure 5).

\section{Conclusion and perspectives}

This study proposes a framework to analyse the hydrodynamic coupling of two slender flags in axial flow when the separation distance is small compared to the flag's length but large compared to its width $(H \ll d \ll L)$. The essential idea of the present model is to account for hydrodynamic coupling by considering the modifications introduced by each flag in the ambient flow seen by its neighbour. The resulting extensions of Lighthill's EBT and LAEBT were used to study the linear and nonlinear dynamics, and in particular the role of hydrodynamic coupling in the synchronization of the two flags' dynamics. In the linear case, flutter instability leads to either in-phase or out-of-phase modes and hydrodynamic coupling appears to destabilize the system. In the nonlinear saturated regime, our simulations show that the flags synchronize after a transient regime. The selected flapping dynamics at long times, and in particular the flags' synchronization, generally corresponds to linear predictions and the linear maps are therefore representative of what should be expected in the nonlinear regime. Out-of-phase dynamics is thus dominant for small values of $M^{*}$, while large $M^{*}$ correspond to a greater sensitivity of the phase to the system's parameters. In addition, in-phase motion is generally expected for small 
$d^{*}$ and out-of-phase motion at larger $d^{*}$. These results are consistent with previous experimental and numerical studies on this topic.

Interestingly, nonlinear selection mechanisms are observed to dominate the linear selection in some cases where in-phase and out-of-phase modes have similar growth rates, and appear to favour in-phase dynamics. The mechanisms of this nonlinear selection are beyond the scope of the present work and should be the focus of further investigation. Understanding such nonlinear selection mechanisms is an important challenge and still an open question in the domain of fluid-structure interactions. The present problem, and its simplified framework, provide an interesting benchmark configuration to investigate this question in greater depth.

Finally, the present framework can easily be extended to account for more than two flags (Michelin \& Llewellyn Smith 2009; Tian et al. 2011b; Udding, Huang \& Sung 2013; Favier, Revell \& Pinelli 2015) and to couple the fluid-solid system to an electric generator as for piezoelectric flags (Michelin \& Doaré 2013; Xia, Michelin \& Doaré 2015).

\section{Acknowledgement}

This work was supported by the French National Research Agency ANR (grant no. ANR-2012-JS09-0017).

\section{REFERENCES}

Abramowitz, M. \& Stegun, I. 1964 Handbook of Mathematical Functions: with Formulas, Graphs, and Mathematical Tables, vol. 55. Courier Corporation.

Alben, S. 2009 Wake-mediated synchronization and drafting in coupled flags. J. Fluid Mech. 641, 489-496.

Banerjee, S., Connell, B. S. H. \& Yue, D. K. P. 2015 Three-dimensional effects on flag flapping dynamics. J. Fluid Mech. 783, 103-136.

CAndelier, F., Boyer, F. \& Leroyer, A. 2011 Three-dimensional extension of Lighthill's largeamplitude elongated-body theory of fish locomotion. J. Fluid Mech. 674, 196-226.

CAndelier, F., Porez, M. \& Boyer, F. 2013 Note on the swimming of an elongated body in a non-uniform flow. J. Fluid Mech. 716, 616-637.

DoAré, O. \& Michelin, S. 2011 Piezoelectric coupling in energy-harvesting fluttering flexible plates: linear stability analysis and conversion efficiency. J. Fluids Struct. 27 (8), 1357-1375.

Eloy, C., Doaré, O., Duchemin, L. \& Schouveiler, L. 2010 A unified introduction to fluid mechanics of flying and swimming at high Reynolds number. Exp. Mech. 50 (9), 1361-1366.

Eloy, C., Kofman, N. \& Schouveiler, L. 2012 The origin of hysteresis in the flag instability. J. Fluid Mech. 691, 583-593.

Eloy, C., Souilliez, C. \& Schouveiler, L. 2007 Flutter of a rectangular plate. J. Fluids Struct. 23 (6), 904-919.

FARnell, D., David, T. \& BARton, D. C. 2004 Coupled states of flapping flags. J. Fluids Struct. 19 (1), 29-36.

Favier, J., Revell, A. \& Pinelli, A. 2015 Numerical study of flapping filaments in a uniform fluid flow. J. Fluids Struct. 53, 26-35.

Giacomello, A. \& PORfiri, M. 2011 Underwater energy harvesting from a heavy flag hosting ionic polymer metal composites. J. Appl. Phys. 109, 084903.

JACKSON, J. D. 1999 Classical Electrodynamics. Wiley.

JiA, L. B., LI, F., YIN, X. Z. \& YIN, X. Y. 2007 Coupling modes between two flapping filaments. J. Fluid Mech. 581, 199-220.

Lighthill, M. J. 1960 Note on the swimming of slender fish. J. Fluid Mech. 9 (02), 305-317.

Lighthill, M. J. 1970 Aquatic animal propulsion of high hydromechanical efficiency. J. Fluid Mech. 44 (02), 265-301. 
Lighthill, M. J. 1971 Large-amplitude elongated-body theory of fish locomotion. Proc. R. Soc. Lond. B 179 (1055), 125-138.

Michelin, S. \& DoARÉ, O. 2013 Energy harvesting efficiency of piezoelectric flags in axial flows. J. Fluid Mech. 714, 489-504.

Michelin, S. \& Llewellyn Smith, S. G. 2009 Linear stability analysis of coupled parallel flexible plates in an axial flow. J. Fluids Struct. 25 (7), 1136-1157.

Schouveiler, L. \& Eloy, C. 2009 Coupled flutter of parallel plates. Phys. Fluids 21 (8), 081703.

Shelley, M. J. \& ZhANG, J. 2011 Flapping and bending bodies interacting with fluid flows. Annu. Rev. Fluid Mech. 43, 449-465.

Singh, K., Michelin, S. \& DE LANGRe, E. $2012 a$ The effect of non-uniform damping on flutter in axial flow and energy harvesting strategies. Proc. R. Soc. Lond. A 468, 3620-3635.

Singh, K., Michelin, S. \& DE LAngre, E. $2012 b$ Energy harvesting from axial fluid-elastic instabilities of a cylinder. J. Fluids Struct. 30, 159-172.

Tian, F.-B., LuO, H., ZhU, L., LiaO, J. C. \& LU, X.-Y. $2011 a$ An efficient immersed boundarylattice Boltzmann method for the hydrodynamic interaction of elastic filaments. J. Comput. Phys. 230, 7266-7283.

Tian, F.-B., LuO, H., ZHU, L. \& LU, X.-Y. $2011 b$ Coupling modes of three filaments in side-by-side arrangement. Phys. Fluids 23, 111903.

UdDING, E., HUANG, W.-X. \& SUNG, H. J. 2013 Interaction modes of multiple flags in a uniform flow. J. Fluid Mech. 729, 563-583.

Xia, Y., Michelin, S. \& DoARÉ, O. 2015 Fluid-solid-electric lock-in of energy-harvesting piezoelectric flags. Phys. Rev. Appl. 3 (1), 014009.

Zhang, J., Childress, S., Libchaber, A. \& Shelley, M. 2000 Flexible filaments in a flowing soap film as a model for one-dimensional flags in a two-dimensional wind. Nature 408 (6814), 835-839.

Zhu, L. \& Peskin, C. S. 2003 Interaction of two flapping filaments in a flowing soap film. Phys. Fluids 15 (7), 1954-1960. 